conrsing over its surface, and presented a yellowish, translucent, semi-gelatinous appearance, not unlike that of the cord itself. Below the symphysis pubis were the two labia majora, widely separated by a tense swelling the size of a walnut, which appeared to be prolapsed bowel. It presented a bright-red mucous surface, which bled slightly on handling, and became more tense when the child cried or strained. From the centre of the swelling sprang a small projection, with its apex pointing upwards, in size and appearance suggesting a penis denuded of cutis, and having at the extremity a small opening, which admitted a probe about a quarter of an inch. There was no anus visible, but fres escaped from a slit-like opening in the lower margin of the tumour, and slightly behind it was a a dimple in the skin, occupying the normal position of the anus. The vaginal opening was to the right of the tumour, enclosed by the labia minora; and the meatus urinarius was placed anterior to, and slightly to the inner side of, the vaginal opening, entirely concealed by a fold of the prolapsed gut. Perfect control was exhibited over both sphincters. In other respects the child appeared to be calthy and well formed. The accompanying sketch will

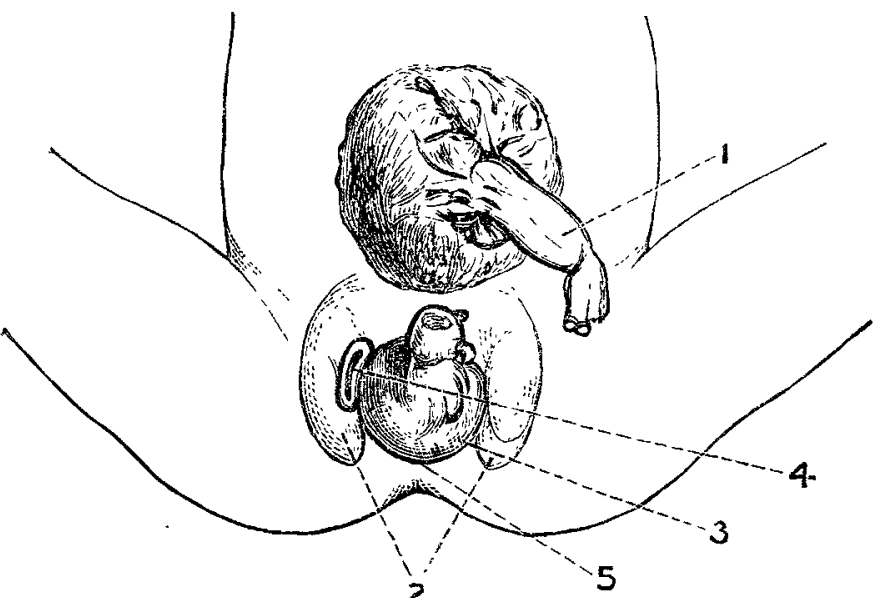

1, Umbilical cord. 2, Lcioia majora. 3, Prolapsed bowel. 4, Vaginal opening. 5, Point from which fæces escaped

give some idea of the appearances just described. The child was fed on milk and barley water, and after about six weeks the opening in the abdominal wall closed by granulation, leaving a small flat cicatrix the size of a threepennypiece. The child survived two months, succumbing to an attack of diarrhoed. It was much emaciated at the time of its death.

Necropsy-On opening the abdominal wall the cavity of the pelvis was found to be occupied entirely by coils of intestine. The bowel bifurcated at the sigmoid flexure into two portions, one of which, bound down tightly by the mesorectum, terminated in the slit-like opening before mentioned; the other, which was about two inches in length, floated freely in the pelvic cavity, and ended in a cul-de-sac, which was pervious up to its extremity, and communicated at its upper end with the continuation of the bowel. In the right iliac fossa was the bladder (which received the right ureter only), the uterus, and the right ovary and its appendages. The left ovary and appendages were attached loosely by connective tissue along the left brim of the pelvis and had yo connexion whatever with the uterus. The left kidney was rudimentary, of the size of a haricot bean, but exhibiting renal structure on section; and the left ureter terminated in a small, white, solid, oval body, the size of a pea, which appeared when divided to cunsist of gland tissue ; it was attached to the left uterine appendages. The right kidney was hypertrophied. Ather previous confinement the mother gave birth to a stillborn male child to all appearances normal.

Walton-on-Thames.

\section{SUPRA-PUBIC CYSTOTOMY IN RETENTION OF URINE - RESULTING FROM CANCER OF} THE VAGINA.

By ARnold CAdDy, F.R.C.S. ENG., SURGEON TO THE CANCER HOSPITAL, LONDON.

The following illustrative case is that of Mrs. Mary Z-aged sixty-five, who was admitted into the Cancer Hospital on Sept. 7th with retention of arine of fifty hours' duration. Her history was that for the last twelve months she has felt something growing in the vagina, and noticed an occasional discharge of blood for the last six months. She has never flooded. She has had difficulty in passing her urine for the last four months. Her father and niece died of cancer of the throat and uterus respectively. On admission the bladder was felt to be distended above the umbilicus. Per vaginam the finger could only be admitted two inches owing to the lumen of the vagina being obstructed by a mass of new growth, which had invaded the anterior wall of the rectum behind and occluded the urethra in front. No urethral orifice was to be seen, and all attempts to pass a catheter consequently falled. Ether was accordingly given at once, and a median incision three inches long was made in the hypogastrium; all the tissues were divided till the bladder was reached, which was punctured, and a finger inserted; some of the urine was allowed to flow out. The bladder wall was then sutured to the skin with eight chromicised catgut sutures. Examination of the floor of the bladder with the finger showed the mucous membrane and the internal urethral orifice to be not yet invaded by the new growth. An indiarubber tube was inserted, and a pad of absorbent cotton wool placed over the wound.-Sept. 8 th : She slept well after the operation, and feels much relieved; the pad of wool over the wound requires changing four times in the twenty-four hours.12th: She got up to-day wearing the drainage-tube.18th: The sutures were removed, and the wound is nearly healed. $-29 \mathrm{th}:$ A Jacques indiarubber catheter was inserted into the supra-pubic opening, and passed through Buckston Browne's shield, retained in position by a belt to be worn permanently.-Oct. 15th: Discharged, improved in health by her stay in hospital. The end of the catheter is connected with a bottle worn from the waist.

This operation is always called for in cases of cancer of the vagina, or cancer of the floor of the bladder where the urethra is involved, and certainly ought to be performed when difficulty in micturition commences, and thus avoiding the distress of extreme and prolonged retention of urine that this patient suffered from. The operation presents no difficulty, and the wound heals very easily if the mucous membrane of the bladder be carefully sutured into apposition with the skin, especially at the upper and lower angles of the wound, whereby all leaking of urine into the cellular tissue is rendered impossible. (For the notes of this case I am indebted to Mr. Cecil Beadles, the senior house surgeon.)

Redcliffe-square, S.W.

\section{A SECOND ATTACK OF VARICELLA AFTER AN} INTERVAL OF TEN DAYS.

\section{By Richard Neale, M.D. LoNd.}

THE recurrence of an attack of varicella is so unusual that its possibility has by some authors been entirely denied. Thomas states that the disease is never followed at once by a complete attack, while Tronsseau is almost the only authority who has seen second attacks of chicken-pox. The following case will prove that it may happen, although undoubtedly very rare.

In the month of October I attended a family of children for chicken-pox, one of whom, a boy aged five years and a half, had all the symptoms of the complaint, numerons vesicles and considerable drowsiness, the attack running its usual course. Ten days after the disappearance of the acute symptoms, and when on the point of being allowed out, a profuse crop of vesicles appeared, which were far more numerons than on the first occasion, and the child again went through all the stages of the original attack, the constitutional disturbance being but slight.

Boundary-road, N.W.

ISOLATION AT LEEDS.-Owing to increase of scarlet fever the Sanitary Committee of the Leeds Corporation are about to ask authority to obtain a new site for a hospital, and to visit some hospitals recently erected by sanitary bodies in other large urban districts. Leeds has long failed to be abreast of many of the large boroughs in respect of really good means for isolating infectious diseases, and it is therefore to be hoped that the needed authority to undertake new hospital buildings will be granted to the com. mittee. 